\title{
Kinetic Energy Estimation in the Nordic System
}

\author{
Mattias Persson \\ Measurement Technology \\ RISE, Research Institutes of Sweden \\ Gothenburg, Sweden
}

\author{
Peiyuan Chen \\ Electric Power Engineering \\ Chalmers University of Technology \\ Gothenburg, Sweden
}

\begin{abstract}
An algorithm for estimating the kinetic energy in the Nordic power system has been presented and compared to the TSOs' estimation based on breaker status. The average error between the TSOs' estimate and the proposed estimate is $0.83 \%$. Furthermore, the standard deviation between the two estimates is $13 \mathrm{GWs}$, about $7 \%$ of the system average kinetic energy. The proposed estimate manages to capture the diurnal variation of the system kinetic energy with a correlation coefficient of 0.94 . The total system kinetic energy in 2015 is estimated considering a possible contribution of kinetic energy from wind turbines during a large power imbalance. Lastly, the Nordic kinetic inertia is estimated for 2025, with and without possible contributions from wind, further increasing the released kinetic energy during a n-1 contingency with $22 \%$.
\end{abstract}

Index Terms-Power system stability, kinetic energy, frequency control, power system.

\section{INTRODUCTION}

Integration of renewable power production and decommissioning of nuclear units causes an increased interest in the kinetic energy available in the synchronous Nordic power systems (NPS). Such a change in generation mix implies a potential reduction in the kinetic energy in the system. The Nordic TSOs have implemented an online inertia estimation tool operating through a SCADA system. The tool keeps track of the breaker positions of synchronously connected generators, their apparent power and their inertia constant. Thereby, the kinetic energy rotating in the system can be estimated. The purpose of this paper is provide a simple estimate of system kinetic energy for future generation scenarios, indicating the risk of too low inertia and possible contributions from wind farms (WFs) utilizing fast frequency response (FFR) algorithms to increase the kinetic energy released during a disturbance. The estimate proposed will present implications due to the change of the Nordic generation mix in 2025 from the one in 2015. Furthermore, the proposed estimate of 2015 will be compared to the estimate implemented by the TSOs.

\section{METHOD}

Production data from ENTSO-E transparency platform are sorted, depending on generation type, into: hydro, $P_{H}$, nuclear, $P_{N}$, thermal, $P_{T}$, wind, $P_{W}$ and solar, $P_{S}$ in MW [1]. The main assumptions of the inertia estimation are as follows:

1) A generator not scheduled for production for a certain hour has its breaker open and does not run in idle operation. This causes the inertia contribution from nonproducing units to be neglected.
TABLE I

ASSUMED H-CONSTANTS OF GENERATORS IN THE NORDIC POWER SYSTEM.

\begin{tabular}{lcccc}
\hline & SE & NO & FI & DK2 \\
\hline \hline$H_{\text {Nuclear }}$ & 6.2 & - & 6.6 & - \\
$H_{\text {Wind }}$ & 0 & 0 & 0 & 0 \\
$H_{\text {Thermal }}$ & 2.9 & 2.5 & 4.4 & 4.5 \\
$H_{\text {Hydro }}$ & 4.5 & 2.9 & 2.8 & - \\
\hline
\end{tabular}

2) All generators, except solar and wind, are working at $\cos (\phi)$ of 0.9 . Solar and wind units are working at unity power factor.

3) Hydro generators are operating at $0.8 \mathrm{pu}$ of the maximum active power rating.

4) All wind turbines are variable speed wind turbines. Thus, small inertia contributions from directly-connected wind turbines, such as induction generator based wind farms, are neglected.

5) All generator units of a certain generation type, i.e. nuclear, hydro, thermal etc. are assigned an average inertia constant for that specific type for each specific country.

6) The contribution from system load inertia e.g. from directly-coupled machines is neglected.

Under these assumptions, the system inertia constant of the Nordic power system (NPS), $H_{\text {sys }}$, can be estimated by

$$
H_{s y s}=\frac{\frac{P_{H-s e} H_{H-s e}}{0.8 \cos \left(\phi_{H}\right)}+\frac{P_{N-f i} H_{N-f i}}{\cos \left(\phi_{N}\right)} \ldots}{\frac{P_{H-s e}}{0.8 \cos \left(\phi_{H}\right)}+\frac{P_{N-f i}}{\cos \left(\phi_{N}\right)} \ldots}
$$

where, $H_{\text {type-country }}$ represents the average inertia constant of each generation type in each country. Combining (1) with the inertia constants found in Table I, the total Nordic system inertia constant, $H_{\text {sys-nordic }}$, can be estimated for all $8760 \mathrm{~h}$ of 2015. Multiplying $H_{\text {sys-nordic }}$ with the system base gives in the kinetic energy in the NPS, $E_{k-s y s-n o r d i c}$ for 2015. Figure 1 presents the variations in $E_{k-s y s-n o r d i c}$ in 2015, reaching its minimum of 117 GWs on 26 July. During a maximum allowed frequency deviation, $f_{\text {nadir }}=49.0 \mathrm{~Hz}$, the kinetic energy released from synchronous generators can be estimated. Being aware of the system inertia constant and its kinetic energy, it is of interest to know the minimum requirements of the two. 


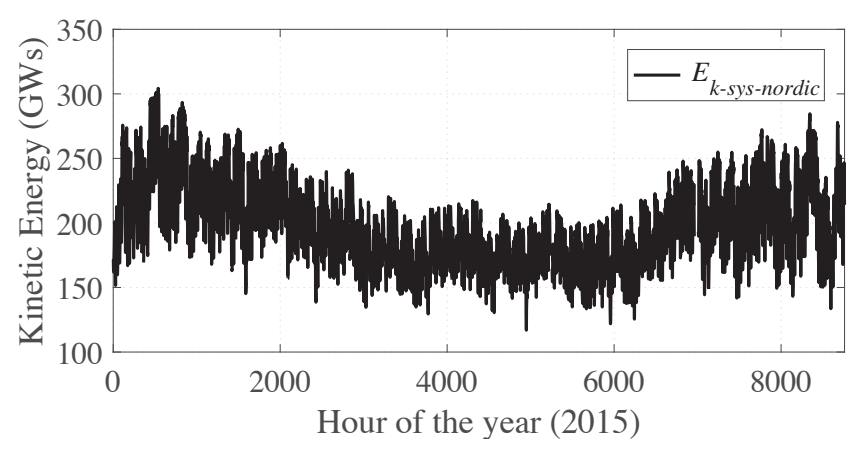

Figure 1. Estimated Nordic kinetic energy during 2015.

\section{A. Minimum level of kinetic energy}

What is the minimum acceptable level of kinetic energy in the system? To answer this question, the authors of [2] presents a simplified calculation of the impact on frequency nadir, $\Delta f_{\text {min }, p u}$, as a function of $H_{\text {sys-nordic }}$ i.e.,

$$
\Delta f_{\text {min }, p u}=\Delta P_{\text {load }, p u} \frac{1}{D}\left(e^{-\frac{D T_{d}}{2 H_{\text {sys }}-\text { nordic }}}-1\right)
$$

where, $\Delta P_{\text {load,pu }}$ represents the change in load or generation, $D$ the load frequency dependence, $H_{\text {sys }}$ the system inertia and $T_{d}$ is time constant for the governors responding with FCRD. Thus, for a lower system inertia (e.g. by integration of variable speed wind turbines (VSWT)) lower frequency nadirs in the system can be expected. Since the minimum frequency allowed is $49.0 \mathrm{~Hz}(-0.02 \mathrm{pu})$, the minimum system inertia can be found by

$$
H_{\text {sys-nordic }}^{\text {min }}=\frac{-T_{d} D}{2\left[\ln \left(\frac{\Delta f_{\min , p u} D}{\Delta P_{\text {load }, p u}}+1\right)\right]}
$$

utilizing a $P_{\text {load }}$ of $1400 \mathrm{MW}$ per system base, $S_{b-s e}$, i.e. the $\mathrm{N}-1$ fault of Sweden (Oskarshamn 3 nuclear power station) and its per unit value is based on the system base, $S_{b-n o r d i c}$. Then, lacking in order to solve (3) are only two items. The frequency dependence, $D$, as well as the time constant for the governor responding to the frequency deviation (assumed to be hydro based) represented by $T_{d}$. The delay time, $T_{d}$, accounts for the governor and turbine response time is estimated to $2.7 \mathrm{~s}$ according to [2]. The load frequency dependence, $D$, for the Nordic power system is mentioned as $400 \mathrm{MW} / \mathrm{Hz}$ in [3]. Thus, its per-unit value varies with the system base, $S_{b-s e}$ assuming a fixed frequency of $50 \mathrm{~Hz}$.

\section{B. Possible wind turbine contribution}

Assuming a wind turbine of a GE 3.6 MW DFIG type presented in [4], as well as considering a controlled inertia response (CIR), $\Delta P_{e}$, provided for a duration of $10 \mathrm{~s}$. The limiting factor for providing the CIR is the minimum rotor speed. This minimum rotor speed decides at what operating point $\Delta P_{e}$ of various magnitudes can be delivered.

Figure 2 presents the results from simulated events with controlled inertia for a fixed duration but with different magnitudes of controlled inertial power, $\Delta P_{e}$, and a different allowed

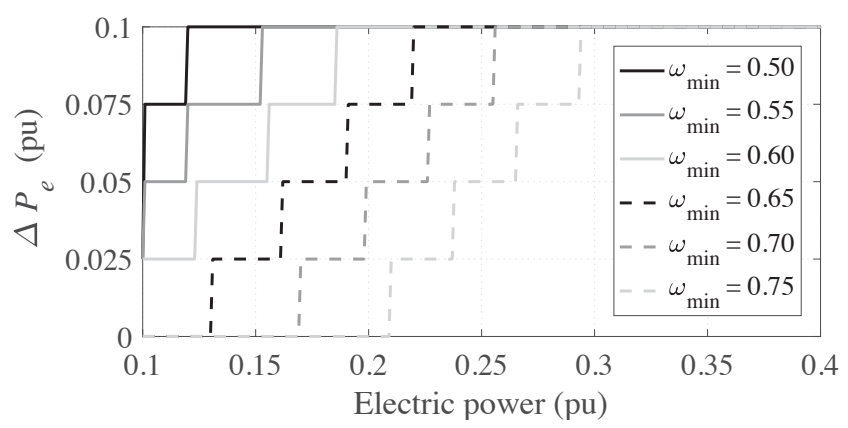

Figure 2. The impact of operating point and the ability to provide a $\Delta P_{e}$ of $0.025,0.05,0.075$ or $0.1 \mathrm{pu}$, when alternating the minimum allowed rotor speed. The duration of the controlled inertia is fixed to $10 \mathrm{~s}$.

TABLE II

Minimum Initial aCtive POWER, $P_{e 0}^{m i n}$ NEEDED IN ORDER to PROVIDE VARIOUS MAGNITUDES OF $\Delta P_{e}$ WITHOUT REACHING A CERTAIN MINIMUM ROTOR SPEED, $\omega_{w t}^{\min }$.

\begin{tabular}{|c|l|l|l|l|}
\hline \multirow{2}{*}{$\omega_{w t}^{\min }(\mathrm{pu})$} & \multicolumn{4}{|c|}{$\Delta P_{e}(\mathrm{pu})$} \\
\cline { 2 - 5 } & 0.025 & 0.05 & 0.075 & 0.1 \\
\hline 0.50 & 0.10 & 0.10 & 0.10 & 0.12 \\
0.55 & 0.10 & 0.10 & 0.12 & 0.15 \\
0.60 & 0.10 & 0.12 & 0.16 & 0.19 \\
0.65 & 0.13 & 0.16 & 0.19 & 0.22 \\
0.70 & 0.17 & 0.20 & 0.23 & 0.26 \\
0.75 & 0.20 & 0.24 & 0.27 & 0.29 \\
\hline
\end{tabular}

minimum rotor speed. The electric power output of the VSWT was swept from 0.1 ( $\left.v_{\text {wind }}=5.21 \mathrm{~m} / \mathrm{s}\right)$ to $1.0 \mathrm{pu}\left(v_{\text {wind }}=\right.$ $11.2 \mathrm{~m} / \mathrm{s})$. The electric power on the $\mathrm{x}$-axis represents the minimum operating point of the VSWT in order to deliver a certain $\Delta P_{e}$ when subject to minimum rotor speed restrictions, $\omega_{w t}^{\min }$.

Assuming that $50 \%$ of the WFs are able to release kinetic energy (between $0.025-0.1$ pu for $10 \mathrm{~s}$ ) depending on operation point, the released energy with and without FFR can be estimated. Figure 3 shows the duration curve of the available kinetic energy that can be released during a power imbalance at any hour of the year. When the WFs provide FFR, an overall improved duration curve can be observed when WFs contribute with FFR.

\section{Nordic system inertia - comparison against TSOs' estimate}

In order to evaluate the behaviour of the inertia estimation it is benchmarked against the Nordic TSOs' inertia estimation tool. Even though it also provides an inertia estimate, its

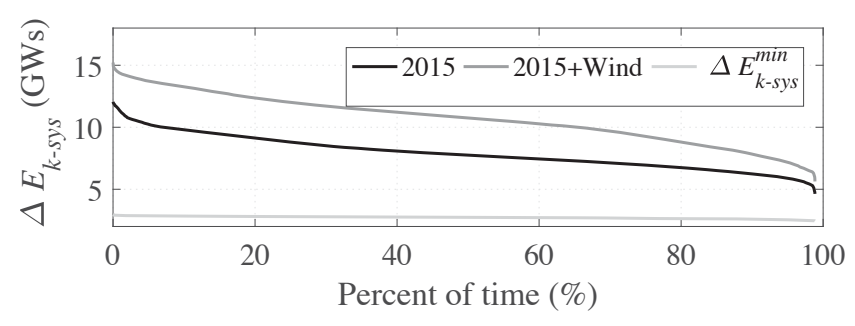

Figure 3. Duration plot of the released kinetic energy with and without 50\% of the aggregated WFs providing FFR during a frequency deviation. 


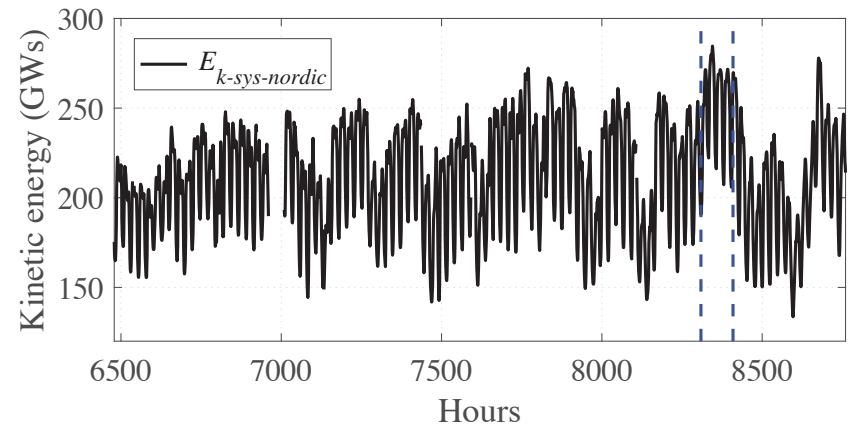

Figure 4. The estimated kinetic energy in the Nordic system during the time period of data from the TSO.

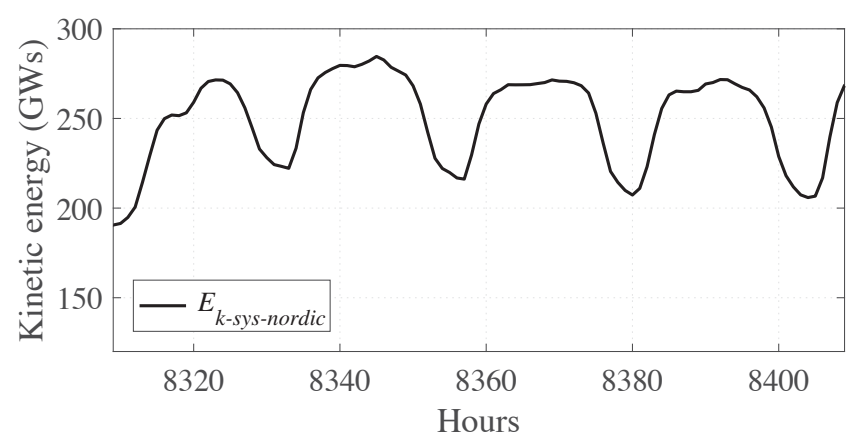

Figure 5. A zoomed in of the estimated kinetic energy in the Nordic system during the time period of maximum estimated kinetic energy.

awareness through the breaker position and an exact value of each generator should come close to that of the real system. The received data covers a time period in the end of 2015 (2015-09-28 - 2015-12-31) is sampled every hour and provides the kinetic energy, $E_{k-T S O-n o r d i c}$, in GWs. Unfortunately, the data is not allowed to be shared as a time-varying signal, only statistical values are allowed to be presented. Figure 4, presents the estimated kinetic energy in the Nordic system, $E_{k-s y s-n o r d i c}$, during the time period of investigation. At 7000 hours, there is noticeable gap in the results due to incoherent production data. Furthermore, the minimum value of the estimated kinetic energy of the period is found to be 133.6 GW, the mean is $210.8 \mathrm{GWs}$ and the maximum value is 284.6 GWs. The area enclosed by vertical bars in Figure 4 represents some of the highest peaks of system inertia during the time period. Figure 5 presents the hourly variations during four days of high inertia estimate enclosed in Figure 4. This location is one of the worst estimates when comparing to the data provided by the TSOs'. The evening and night times are almost identical, the highest $E_{k-s y s-n o r d i c}$ observed is $284.6 \mathrm{GWs}$, this is an overestimation compared to $E_{k-T S O-n o r d i c}$ of $7 \%$. The main reason for this is believed to come from the aggregation of $P_{\text {Thermal-fi }}$. This contains production data from gas, peat, hard coal and oil units. All aggregated with an inertia constant, $H_{\text {Thermal-fi }}$. During these variations, the Finnish aggregated thermal varies the most among the Nordic countries, especially the Finnish peat, gas and hard coal. This

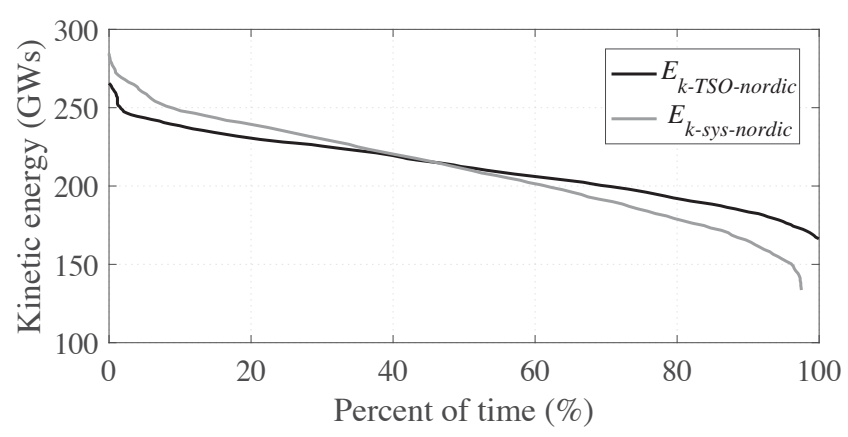

Figure 6. The duration curve of the TSO estimate, $E_{k-T S O-n o r d i c}$ and the proposed estimate $E_{k-s y s-n o r d i c}$.

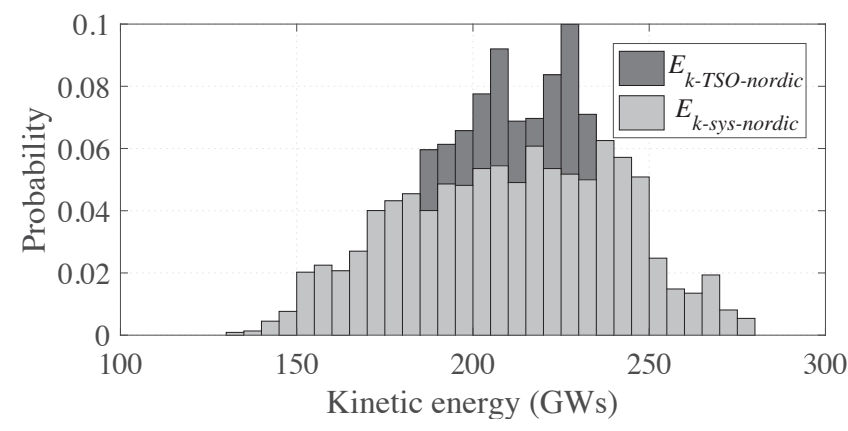

Figure 7. Probability distribution of the two estimates during 3 months of data in 2015.

aggregation is believed to have the greatest impact on the error. Figure 6 presents the duration curves of the two estimates. The lack of production data causes the slightly altered shape of the duration curve for $E_{k-s y s-n o r d i c}$. $E_{k-s y s-n o r d i c}$ is mainly lower than the TSOs' estimate mainly during night times, rarely under $E_{k-T S O-n o r d i c}$ during day time. This is indicated in the increased max and minimum of $E_{k-s y s-n o r d i c}$ in Figure 6. The average error between the TSOs' estimate and the proposed estimate is $0.8 \%$. Furthermore, the standard deviation between $E_{k-T S O-n o r d i c}$ and $E_{k-s y s-n o r d i c}$ is 13 GWs, about $7 \%$ of the TSOs' estimate of the average kinetic energy. Figure 7 present the probability of the different kinetic energy estimates, further indicating the increased spread of $E_{k-s y s-n o r d i c}$. Each bin of the histogram is $5 \mathrm{GWs}$ wide starting at $130 \mathrm{GWs}$ and ending at $280 \mathrm{GWs}$. The overall shape of the two estimate is very similar. One way of evaluating this is by the use of correlation coefficients. The linear correlation coefficient is determined by

$$
r(A, B)=\frac{1}{N-1} \sum_{i=1}^{N}\left(\frac{A_{i}-\mu_{A}}{\sigma_{A}}\right)\left(\frac{B_{i}-\mu_{B}}{\sigma_{B}}\right)
$$

Where, $r(A, B)$ is the correlation coefficient between variable $\mathrm{A}$ and $\mathrm{B}, A_{i}$ and $B_{i}$ is the value of the $i^{\text {th }}$ observation, $\mu$ and $\sigma$ is the mean value and the standard deviation respectively and $N$ is the number of total observations[5]. Solving (4) for $r\left(E_{k-T S O-n o r d i c}, E_{k-s y s-n o r d i c}\right)$ results in a value of 0.94 , 
TABLE III

CURRENT AND EXPECTED INSTALLED CAPACITY OF NUCLEAR AND WIND TURBINES IN THE NORDIC POWER SYSTEM IN 2015 AND 2025 (IN BOLD).

\begin{tabular}{lllll}
\hline $\begin{array}{l}\text { Installed } \\
\text { power }\end{array}$ & $\begin{array}{l}\text { SE [7] } \\
(\mathrm{MW})\end{array}$ & $\begin{array}{l}\text { NO [8] } \\
(\mathrm{MW})\end{array}$ & $\begin{array}{l}\text { FI [9], [10], [11] } \\
(\mathrm{MW})\end{array}$ & $\begin{array}{l}\text { DK2 [12] } \\
(\mathrm{MW})\end{array}$ \\
\hline \hline Nuclear & $\mathbf{6 7 0 0 / 9 5 2 8}$ & - & $\mathbf{5 5 4 9 / 2 7 4 9}$ & - \\
Wind & $\mathbf{1 0 5 0 0 / 5 4 2 0}$ & $\mathbf{3 2 0 7} / 873$ & $\mathbf{3 9 0 0} / 1005$ & $\mathbf{2 6 0 6} / 1036$ \\
\hline
\end{tabular}

indicating a high correlation between the two, since a value of 1 would indicate a completely linear relationship.

\section{FUTURE SCENARIO - 2025}

Except for the reduction in power system inertia in the Swedish system mainly due to wind integration, several other activities are anticipated in the Nordic synchronous area by 2025 . The system development concerns especially the frequency stability of the system. The current largest power imbalance of Oskarshamn 3 is 1400 MW, i.e. the system dimensioning fault, and it is not expected to increase. The new Finnish Olkiluoto nuclear power plant has a largest unit of $1600 \mathrm{MW}$. However, a $300 \mathrm{MW}$ load is also connected in parallel to this unit. If this nuclear unit is tripped, the load will also be tripped at the same time. Thus, the $\mathrm{N}-1$ in this case amounts to only $1300 \mathrm{MW}$ seen from the grid side [6]. Furthermore, Finland's parliament has authorized the construction of another nuclear unit of 1200 MW, Hanhikivi in Pyhäjoki, which is expected to be completed in 2024. These two Finnish nuclear units almost compensate for the dismantling of the Oskarshamn unit 1 in Sweden, of $1100 \mathrm{MW}$ and Ringhals unit 1 and unit 2, in Sweden, of $1750 \mathrm{MW}$ in total [7].

Table III summarizes the installed capacities of the generation of interest nuclear and wind turbines in the Nordic power system in 2015 and 2025. Utilizing the known system changes based on Table III, the generation mix can be observed for 2025 and hence the system's kinetic energy can be estimated, as well as possible contributions from future WFs.

Some added assumptions for this subsection include:

1) Inertia constants of new nuclear units in Finland are similar to the current ones.

2) No hydro or thermal expansion in the Nordic region.

3) Same production patterns as 2015 scaled by Table III

4) Increased wind power is covered by an increased load and increased export.

Following the similar procedure as Section II the system kinetic energy in 2025 can be estimated.

Figure 8 shows the estimated system kinetic energy, $E_{k-s y s-n o r d i c}$, in 2025 and 2015. It is clear that the two are similar. This similarity is partly due to the fact that the average Finnish nuclear inertia constant is larger than the

${ }^{1}$ [8] presents $2080 \mathrm{MW}$ for year 2020. This value is then linearly extrapolated from 2015 to 2025, resulting an approximation of $3207 \mathrm{MW}$ in 2025

${ }^{2}[10]$ presents an increase to $9 \mathrm{TWh}$ wind power in 2025 , [11] presents data for 2015 of $1005 \mathrm{MW}$, producing $2.3 \mathrm{TWh}$. Using this energy to scale production to get about $3900 \mathrm{MW}$ assuming similar wind conditions as in 2015 .

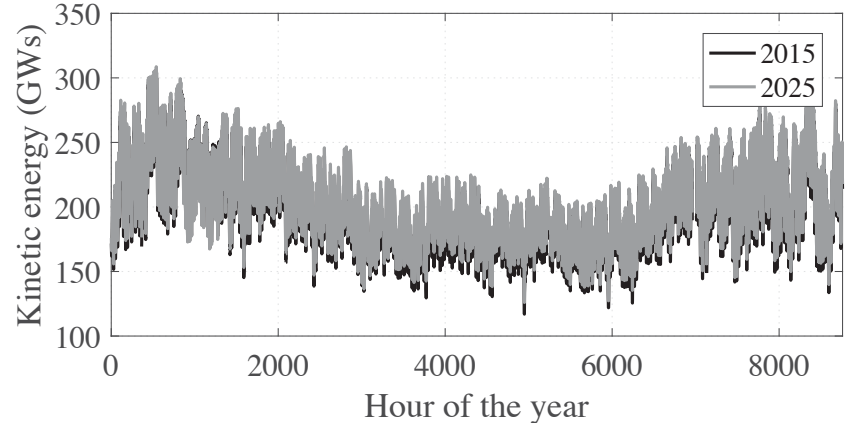

Figure 8. Estimated kinetic energy in 2025 compared to 2015.

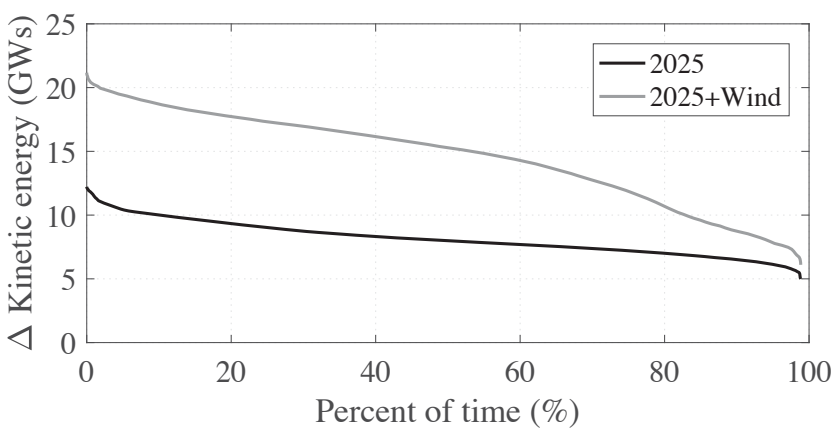

Figure 9. Estimated duration of released kinetic energy with and without $50 \%$ of aggregated wind farms responding with FFR during a frequency deviation down to $49.0 \mathrm{~Hz}$ during 2025.

Swedish one. Therefore, the nuclear installed capacity in 2025 is replaced with a higher average inertia constant. This higher average cause the total inertia constant to increase, even though there is a negative contribution due to wind integration. This is especially true under the assumption that the load and/or export covers the generation increase from 2015 to 2025. The added wind power could possibly provide even larger contributions than observed in Figure 3 seen to the released kinetic energy in 2025 during a frequency deviation since the increased installed capacity.

As expected the minimum kinetic energy released is increased in Figure 9 by $22 \%$. Furthermore, for $50 \%$ of the time, the increase in released kinetic energy is $90 \%$ when considering the contribution with FFR from an aggregated wind farm in 2025.

\section{Discussion}

An estimate of the kinetic energy in the system could, combined with knowledge of reserves, give incentives on the balancing market similar to pay-for-performance structures. Better, more highly qualitative frequency controlling objects could then be scheduled to ensure the frequency stability. Ensuring the frequency stability could give TSOs a variable tool for estimating their need for frequency reserves of various speeds and quality. Therefore, inertia estimation tools could prove to reduce cost for frequency reserves during hours of high kinetic energy or a small n-1 contingency in the system. 
If utilized in a smaller sub part of a power system a kinetic energy estimate could indicate whether an islanded operation is feasible, whether the sub system could manage its n-1 contingency and recommendations for reserves.

Lastly, if the kinetic energy is deemed to low, inertia could be connected to the power system by simply rotating non producing generators. However, no incentives for such operation are available in the Nordic system today, even though it on some occasions are done for the sake of voltage regulation. Perhaps this is just a matter of time?

\section{Conclusions}

A tool for the estimation of kinetic energy in the Swedish and Nordic system is proposed. The estimate could be used after the clearing of the day ahead to provide good insight to the upcoming operation challenges facing the TSO.

The trends for the Nordic system kinetic energy for 2015 and 2025 were estimated. The 2025 scenario showed that, under the assumption that all added generation is consumed by a load increase, the kinetic energy would slightly increase after the replacement of Swedish nuclear units by Finnish nuclear. This increase was mainly due to the larger nuclear inertia constant in Finnish plants. However, if the load and import/export were assumed to be constant in 2025, a slight decrease in the system kinetic energy could be observed.

The estimation algorithm was benchmarked against the TSOs' algorithm for 3 months in 2015. The comparison show a high correlation factor of 0.94 , an average error of $0.8 \%$ and a standard deviation of $13 \mathrm{GWs}$ ( $7 \%$ of the average kinetic energy). The method can be used by the TSOs or the providers of ancillary service to estimate the kinetic energy in the system, and schedule reserves accordingly.

Lastly, the very pessimistic view on solar and wind expansion set by the TSOs in their development plan is likely to alter. However, the suggested method could include FFR from batteries, HVDC links or other more aggressive scenarios for renewable integration to show their impact on the inertia in future power systems.

\section{REFERENCES}

[1] ENTSO-E. (2017) ENTSO-E transperency platform. [Online]. Available: https://transparency.entsoe.eu/

[2] N. Ullah, T. Thiringer, and D. Karlsson, "Temporary primary frequency control support by variable speed wind turbines; potential and applications," IEEE Transactions on Power Systems, vol. 23, no. 2, pp. 601-612, May 2008

[3] ENTSO-E, Nordic balancing philosophy, Jun, 2016

[4] N. W. Miller, Modeling of GE wind turbine-generators for grid studies - V4.2, June 2008.

[5] R. A. S. Fisher, Statistical methods for research workers, 13th ed. Edinburgh : Oliver and Boyd, 1958.

[6] Svenska kraftnät, Statnett, Fingrid and Energinet.dk, Challenges and Opportunities for the Nordic Power System, Aug 2016.

[7] Svenska kraftnät, Network development plan 2016-2025 - A Ten-year Plan for the Swedish National Grid. Svenska kraftnät, 2016-11-26.

[8] ENTSO-E, TYNDP 2016 scenario development report. ENTSO-E, May 2015.

[9] Radiation and Nuclear Safety Authority (STUK). (2017) Nuclear power plants in finland. [Online]. Available: http://www.stuk.fi/web/en/topics/nuclear-power-plants/nuclearpower-plants-in-finland
[10] Hannele Holttinen and Esa Peltola, VTT Technical Research Centre of Finland, Finland, IEA Annual report - 20. Finland, 2017.

[11] VTT Technical Research Centre of Finland, Wind energy statistics in Finland, 2017.

[12] Energinet.dk, Energinet.dk's analysis assumptions 2014-2035, Update September 2014. Energinet.dk, Aug 2014. 